\title{
Nonlocal dynamo waves in a turbulent shear flow
}

\author{
G. Rüdiger ${ }^{1}$ and V. Urpin ${ }^{1,2}$ \\ 1 Astrophysikalisches Institut Potsdam, An der Sternwarte 16, 14482 Potsdam, Germany \\ 2 A.F. Ioffe Institute of Physics and Technology, 194021 St. Petersburg, Russia \\ Received 22 September 2000 / Accepted 12 January 2001
}

\begin{abstract}
The turbulent dynamo action in a shear flow is considered by making use of a quasilinear approximation and neglecting the back-reaction of a generated magnetic field on turbulence. The shear can stretch turbulent magnetic field lines in such a way that turbulent motions may become suitable for the generation of a large-scale magnetic field even in the absence of any stratification. There is no $\alpha$-effect present in our computations. The nonlocal integral representation for the mean electromotive force is derived, which is valid even if the turbulent length scale is comparable to that of the mean field. The basic result is that the presence of shear changes the type of the equation governing the mean magnetic field so that the latter indeed can be generated even in the absence of rotation or large-scale stratification of turbulence. To this end, however, if the turbulence field has a monotonously falling ("turbulence-type") spectrum, a rather strong shear is needed. For Kepler disks the instability condition reads $\tau_{\text {corr }}>2 \tau_{\text {rot }} / \pi$, which might be fulfilled in the transition layers between star and disk. A system, on the other hand, consisting of random waves, large-scale magnetic fields and mean-field shear flow can never be stable.
\end{abstract}

Key words. magnetohydrodynamics - stars: magnetic fields - interstellar medium: magnetic fields - accretion, accretion discs

\section{Introduction}

It is generally believed that turbulent fluid motions showing a lack of mirror symmetry are most suitable for a large scale dynamo action (see, e.g., Moffatt 1978; Parker 1979; Krause \& Rädler 1980). In the cases of astrophysical interest, departures from mirror symmetry are usually provided by rotation. The Coriolis force distorts turbulent motions in such a way that the resulting mean electromotive force has a component proportional to the mean magnetic field (the alpha-effect) which can be responsible for a dynamo action.

However, apart from the alpha-effect, there exists another mechanism for the generation of a large-scale magnetic field in turbulent fluids. Recently, it has been argued by Urpin (1999a,b) that a qualitatively different mechanism can operate in a fluid in the presence of shear. Shear stretches turbulent magnetic field lines in the direction of mean motion, and this stretching leads to the generation of an additional component of the mean electromotive force which is proportional to the product of spatial derivatives of the mean velocity and magnetic field. The presence of such terms in the mean electromotive force has been shown by Hoyng (1985) who considered the case of differential rotation with $\Omega=\Omega(z)$ and used a Lagrangian representation for the mean velocity and an Eulerian one for the

Send offprint requests to: G. Rüdiger, e-mail: gruediger@aip.de turbulent velocity. Numerical simulations of the dynamo action in a shear-flow turbulence also indicate the presence of shear-driven terms (Pipin et al. 2001). Contrary to the conventional alpha-dynamo, the shear-driven dynamo can generate a large-scale magnetic field even if there is no density stratification (or stratification of turbulence intensity) in the fluid.

In the present paper, the properties of a shear-driven dynamo are considered avoiding the two-scale approximation used by Urpin (1999a,b) and Hoyng (1985). We derive the integral expression for the mean electromotive force which is valid even if the length scales of turbulence and mean field are comparable. In accordance with our results, the mean electromotive force at some point is determined by the spatial distribution of the mean magnetic field at specific instances of time. The induction equation with such an electromotive force has a solution corresponding to nonlocal dynamo waves which can be unstable under certain conditions. These waves represent the mean field which is two-dimensional and periodic in the direction of a mean flow. As a tool for our consideration, a simple kinematic model is used, neglecting the influence of the generated magnetic field on turbulence.

\section{The mean turbulent electromotive force}

The magnetic field $\boldsymbol{B}$ and the velocity $\boldsymbol{u}$ are usually written as sums of their mean and fluctuating parts, 
$\boldsymbol{B}=\overline{\boldsymbol{B}}+\boldsymbol{B}^{\prime}$ and $\boldsymbol{u}=\overline{\boldsymbol{u}}+\boldsymbol{u}^{\prime}$, where $\overline{\boldsymbol{B}}$ and $\overline{\boldsymbol{u}}$ are the mean quantities. The mean induction equation reads,

$$
\frac{\partial \overline{\boldsymbol{B}}}{\partial t}=\nabla \times(\overline{\boldsymbol{u}} \times \overline{\boldsymbol{B}})+\eta \Delta \overline{\boldsymbol{B}}+\nabla \times \mathcal{E}
$$

where $\eta$ is the magnetic viscosity and $\mathcal{E}=\left\langle\boldsymbol{u}^{\prime} \times \boldsymbol{B}^{\prime}\right\rangle$ is the mean electromotive force (EMF); $\langle\ldots\rangle$ denotes ensemble averaging. We consider $\mathcal{E}$ in a quasilinear approximation. In this approximation, mean quantities are governed by equations including non-linear effects in fluctuating terms, while the linearised equation are used for the fluctuating quantities (see, e.g., Krause \& Rädler 1980).

The linearised induction equation for the fluctuating magnetic field reads

$$
\frac{\partial \boldsymbol{B}^{\prime}}{\partial t}=\operatorname{rot}\left(\overline{\boldsymbol{u}} \times \boldsymbol{B}^{\prime}\right)+\operatorname{rot}\left(\boldsymbol{u}^{\prime} \times \overline{\boldsymbol{B}}\right)+\eta \Delta \boldsymbol{B}^{\prime}
$$

Consider a plane incompressible Couette flow with the mean velocity given by $\overline{\boldsymbol{u}}=V(x) \boldsymbol{e}_{y}$ where $x, y$ and $z$ are the Cartesian coordinates; $\boldsymbol{e}_{x}, \boldsymbol{e}_{y}$, and $\boldsymbol{e}_{z}$ are the unit vectors. Shear is assumed to be linear, $V(x)=V_{0}+x \mathrm{~d} V / \mathrm{d} x$. Substituting this expression into (2), we have

$$
\frac{\partial \boldsymbol{B}^{\prime}}{\partial t}+V(x) \frac{\partial \boldsymbol{B}^{\prime}}{\partial y}-\boldsymbol{e}_{y} B_{x}^{\prime} \frac{\mathrm{d} V}{\mathrm{~d} x}-\eta \Delta \boldsymbol{B}^{\prime}=\boldsymbol{A}^{\prime},
$$

with $\boldsymbol{A}^{\prime}=(\overline{\boldsymbol{B}} \cdot \nabla) \boldsymbol{u}^{\prime}-\left(\boldsymbol{u}^{\prime} \cdot \nabla\right) \overline{\boldsymbol{B}}$. We assume that the magnetic Reynolds number is very large for turbulent motions, thus we can neglect the dissipative term (except the cases when we need to know the sign of this term which can be important to choose properly the integration path for calculating Fourier integrals).

We solve Eq. (3) by use of a Fourier transformation in $\boldsymbol{r}$ and $t$. In the Appendix A the mathematical details of this procedure are demonstrated in detail. The solution for a fluctuating magnetic field then can be written as

$$
\boldsymbol{B}^{\prime}(\boldsymbol{r}, t)=\int_{-\infty}^{+\infty} \frac{\mathrm{d} \omega \mathrm{d} \boldsymbol{k}}{i \omega_{1}} \mathrm{e}^{i\left(\omega+k_{y} V\right) t-i \boldsymbol{k} \boldsymbol{r}}\left(\hat{\boldsymbol{A}}-\frac{i}{\omega_{1}} \frac{\mathrm{d} V}{\mathrm{~d} x} \hat{A}_{x} \boldsymbol{e}_{y}\right)
$$

where $\omega_{1}=\omega-i 0 ; \pm 0$ denotes a positive (or negative) contribution of small dissipative terms proportional to $\eta$. The result looks like a Doppler-shifted Fourier transform with the frequency $\omega+k_{y} V(x)$.

Substituting the Fourier transformation of $\boldsymbol{u}^{\prime}(\boldsymbol{r}, t)$ and the above expression for $\boldsymbol{B}^{\prime}(\boldsymbol{r}, t)$ into the definition of $\mathcal{E}$, we have

$$
\begin{aligned}
& \mathcal{E}=i \int \frac{\mathrm{d} \omega^{\prime} \mathrm{d} \boldsymbol{k}^{\prime}}{(2 \pi)^{4}} \frac{\mathrm{d} \omega \mathrm{d} \boldsymbol{k}}{\omega_{1}} \mathrm{~d} \omega^{\prime \prime} \mathrm{d} \boldsymbol{k}^{\prime \prime} \mathrm{d} \boldsymbol{r}^{\prime} \mathrm{d} t^{\prime}\left\langle\hat{\boldsymbol{u}}\left(\boldsymbol{k}^{\prime}, \omega^{\prime}\right) \times \boldsymbol{M}\right\rangle \\
& \times \mathrm{e}^{i\left\{\left(\omega+\omega^{\prime}\right) t-\left(\boldsymbol{k}+\boldsymbol{k}^{\prime}\right) \boldsymbol{r}-\left(\omega-\omega^{\prime \prime}\right) t^{\prime}+\left(\boldsymbol{k}-\boldsymbol{k}^{\prime \prime}\right) \boldsymbol{r}^{\prime}+k_{y}\left[V(x) t-V\left(x^{\prime}\right) t^{\prime}\right]\right\}}
\end{aligned}
$$

with

$$
\begin{aligned}
& \boldsymbol{M}=i\left(\boldsymbol{k}^{\prime \prime} \cdot \overline{\boldsymbol{B}}\right) \hat{\boldsymbol{u}}\left(\boldsymbol{k}^{\prime \prime}, \omega^{\prime \prime}\right)+\left(\hat{\boldsymbol{u}}\left(\boldsymbol{k}^{\prime \prime}, \omega^{\prime \prime}\right) \cdot \nabla^{\prime}\right) \overline{\boldsymbol{B}}\left(\boldsymbol{r}^{\prime}, t^{\prime}\right) \\
& -\frac{i \mathrm{~d} V}{\omega_{1} \mathrm{~d} x}\left(i\left(\boldsymbol{k}^{\prime \prime} \overline{\boldsymbol{B}}\right) \hat{u}_{x}\left(\boldsymbol{k}^{\prime \prime}, \omega^{\prime \prime}\right)+\hat{\boldsymbol{u}}\left(\boldsymbol{k}^{\prime \prime}, \omega^{\prime \prime}\right) \cdot \nabla^{\prime} \bar{B}_{x}\left(\boldsymbol{r}^{\prime}, t^{\prime}\right)\right) \boldsymbol{e}_{y}
\end{aligned}
$$

For simplicity we assume that turbulence is locally isotropic and homogeneous with the correlation tensor given by

$$
\begin{aligned}
\left\langle\hat{u}_{i}(\boldsymbol{k}, \omega) \hat{u}_{j}\left(\boldsymbol{k}^{\prime}, \omega^{\prime}\right)\right\rangle= & \frac{1}{3} q(\boldsymbol{k}, \omega)\left(\delta_{i j}-k_{i} k_{j} / k^{2}\right) \\
& \times \delta\left(\boldsymbol{k}+\boldsymbol{k}^{\prime}\right) \delta\left(\omega+\omega^{\prime}\right),
\end{aligned}
$$

where $q(\boldsymbol{k}, \omega)$ is the spectral function (see, e.g., Rüdiger 1989). Of course, shear can affect the correlation properties and, probably, turbulence is not isotropic and homogeneous in real conditions; this effect, however, is neglected here.

With the results given in Appendix B the final expression for the mean EMF reads

$$
\begin{aligned}
\mathcal{E}= & -\int_{-\infty}^{t} \mathrm{~d} t^{\prime} F\left(t-t^{\prime}\right)\left\{\nabla^{\prime} \times \overline{\boldsymbol{B}}\left(\boldsymbol{r}^{\prime}, t^{\prime}\right)\right. \\
& \left.-\left(t-t^{\prime}\right) \frac{\mathrm{d} V}{\mathrm{~d} x} \boldsymbol{e}_{y} \times \nabla^{\prime} \bar{B}_{x}\left(\boldsymbol{r}^{\prime}, t^{\prime}\right)\right\}\left.\right|_{\boldsymbol{r}^{\prime}=\boldsymbol{r}-\boldsymbol{V}(x)\left(t-t^{\prime}\right)},
\end{aligned}
$$

where

$F(t)=\frac{2}{9} \int q(\boldsymbol{k}, \omega) \mathrm{e}^{(i \omega-i 0) t} \mathrm{~d} \boldsymbol{k} \mathrm{d} \omega$

contains the spectrum $q$ of the turbulence. Note that deriving this equation we did not assume that the turbulent scale $\ell_{\text {corr }}$ is small compared to that of the mean field. As a result, the mean EMF (7) is nonlocal. The expression for $\mathcal{E}$ does not contain the component proportional to $\overline{\boldsymbol{B}}$ which was typical for the alpha-effect. Since we derived Eq. (7) in a quasilinear approximation, it also does not contain a term proportional to $\boldsymbol{\Omega}$ without any inclusion of the mean magnetic field ("cross-helicity effect"), as in Brandenburg \& Urpin (1998) and Blackman (2000) ${ }^{1}$.

Only magnetic gradients exist in relation (7) for the mean EMF and the mean magnetic field. In the highconductivity limit $(\eta \rightarrow 0)$, the kernel (8) equals the time correlation function $\left\langle u_{i}^{\prime}(\boldsymbol{x}, t) u_{i}^{\prime}(\boldsymbol{x}, t+\tau)\right\rangle$ as a function of $\tau$, which is well-known from experiments. If - as for white noise - it is a delta-function, then (7) turns into the simple and local relation $\mathcal{E} \simeq-\operatorname{rot} \bar{B}$.

\section{The dispersion equation for dynamo waves}

Consider the behaviour of the mean field governed by the EMF (7). The $x$-component of the induction Eq. (1) reads for a plane Couette flow

$$
\frac{\partial \bar{B}_{x}}{\partial t}+V(x) \frac{\partial \bar{B}_{x}}{\partial y}-\eta \Delta \bar{B}_{x}=\operatorname{rot}_{x} \mathcal{E} .
$$

Generally, the shear-driven dynamo mechanism allows a generation of two dimensional magnetic fields with $\bar{B}_{z}=$ 0 . The horizontal field, $\bar{B}_{y}$, can then be calculated from the condition $\operatorname{div} \overline{\boldsymbol{B}}=0$. Substituting the expression (7) for $\mathcal{E}$

\footnotetext{
${ }^{1}$ It represents an equatorially symmetric current system.
} 
into (9) and introducing the new variable of integration, $\tau=t-t^{\prime}$, we obtain the integral equation

$$
\begin{aligned}
& \frac{\partial \bar{B}_{x}}{\partial t}+V \frac{\partial \bar{B}_{x}}{\partial y}-\eta \Delta \bar{B}_{x}=\int_{0}^{\infty} F(\tau)\left[\left(\frac{\partial^{2}}{\partial y^{\prime 2}}-\tau \frac{\mathrm{d} V}{\mathrm{~d} x} \frac{\partial^{2}}{\partial x^{\prime} \partial y^{\prime}}\right)\right. \\
& \left.\bar{B}_{x}\left(\boldsymbol{r}^{\prime}, t-\tau\right)-\frac{\partial^{2}}{\partial x^{\prime} \partial y^{\prime}} \bar{B}_{y}\left(\boldsymbol{r}^{\prime}, t-\tau\right)\right]\left.\right|_{\boldsymbol{r}^{\prime}=\boldsymbol{r}-\boldsymbol{V} \tau} \mathrm{d} \tau
\end{aligned}
$$

for the mean magnetic field. Here we treat the properties of dynamo waves which can exist in a shear flow between two planes, $x=0$ and $x=\mathrm{d}$. We assume that the bottom plane, $x=0$, is at rest; thus $V_{0}=0$ and the flow is given by $V(x)=x \mathrm{~d} V / \mathrm{d} x$. For a very high magnetic Reynolds number one can neglect the dissipative term on the LHS of (10). Since the coefficients of (10) do not depend on $y$, its solution can be written as $\bar{B}_{x}=\beta(x) \mathrm{e}^{\gamma t-i K_{y} y}$, where $K_{y}$ is the wave vector in the $y$-direction and $\gamma$ is the growth rate. Then, the equation for $\beta(x)$ reads

$$
\begin{aligned}
\left(\gamma-i K_{y} V\right) \beta(x)= & \int_{0}^{\infty} F(\tau) \mathrm{e}^{\left(i K_{y} V(x)-\gamma\right) \tau}\left(\frac{\partial^{2}}{\partial x^{2}}-K_{y}^{2}\right. \\
& \left.+i \tau \frac{\mathrm{d} V}{\mathrm{~d} x} K_{y} \frac{\partial}{\partial x}\right) \beta(x) \mathrm{d} \tau
\end{aligned}
$$

With a simplification presented in Appendix C, Eq. (11) transforms into

$$
\begin{aligned}
\left(\gamma-i K_{y} V(x)\right) \beta= & \frac{2}{9} \int q \mathrm{e}^{(i \omega-\gamma) \tau}\left[\left(\frac{\mathrm{d}^{2}}{\mathrm{~d} x^{2}}-K_{y}^{2}\right) \beta\right. \\
& \left.+i \tau \frac{\mathrm{d} V}{\mathrm{~d} x} K_{y} \frac{\mathrm{d} \beta}{\mathrm{d} x}\right] \mathrm{d} \tau \mathrm{d} \boldsymbol{k} \mathrm{d} \omega .
\end{aligned}
$$

For unstable waves $\operatorname{Re}(\gamma)>0$, therefore the integral over $\mathrm{d} \tau$ can easily be calculated. Since for a steady state turbulence $q(\boldsymbol{k},-\omega)=q(\boldsymbol{k}, \omega)$, only the real parts of integrals in (12) are nonvanishing. Then, Eq. (12) can be rewritten as

$\eta_{\mathrm{T}} \frac{\mathrm{d}^{2} \beta}{\mathrm{d} x^{2}}+i K_{y} \frac{\mathrm{d} V}{\mathrm{~d} x} \ell_{2}^{2}(\gamma) \frac{\mathrm{d} \beta}{\mathrm{d} x}-\left[\gamma+\eta_{\mathrm{T}} K_{y}^{2}-i K_{y} V(x)\right] \beta=0$,

where $\eta_{\mathrm{T}}=\gamma \ell_{1}^{2}(\gamma)$ is the turbulent magnetic diffusivity, and $\ell_{1}(\gamma)$ and $\ell_{2}(\gamma)$ are characteristic turbulent length scales, i.e.

$\ell_{1}^{2}=\frac{2}{9} \int \frac{q}{\omega^{2}+\gamma^{2}} \mathrm{~d} \boldsymbol{k} \mathrm{d} \omega, \ell_{2}^{2}=\frac{2}{9} \int \frac{\left(\omega^{2}-\gamma^{2}\right) q}{\left(\omega^{2}+\gamma^{2}\right)^{2}} \mathrm{~d} \boldsymbol{k} \mathrm{d} \omega$.

Note that the latter expression can possess both signs.

\section{Solution of the dispersion equation}

To solve Eq. (13) two boundary conditions are needed. We consider the simplest case assuming the field to be vanishing both at the top and bottom surfaces of a shear flow, i.e. $\beta=0$ at $x=0$ and $x=d$. Note that the main conclusions of our paper are not sensitive to the particular choice of boundary conditions.
It is convenient to make a substitution,

$$
\beta(x)=\mathrm{e}^{-i K_{y} V^{\prime} \ell_{2}^{2}(\gamma) / 2 \eta_{\mathrm{T}}} \hat{\beta}(x),
$$

into (13). Then

$$
\frac{\mathrm{d}^{2} \hat{\beta}}{\mathrm{d} x^{2}}-\left[\frac{\gamma-i K_{y} V(x)}{\eta_{\mathrm{T}}}+K_{y}^{2}-\frac{K_{y}^{2} \ell_{2}^{4}(\gamma)}{4 \eta_{\mathrm{T}}^{2}}\left(\frac{\mathrm{d} V}{\mathrm{~d} x}\right)^{2}\right] \hat{\beta}=0 .
$$

Since $V(x)$ is only a linear function of $x$, the general solution of this equation can be obtained in terms of the Bessel functions of the order $1 / 3$. However, in some particular cases the solution can be substantially simplified. For example, if

$$
\left(K_{y} d\right)\left(\frac{\ell_{2}}{d}\right)^{4} \frac{d^{2}}{2 \eta_{\mathrm{T}}} \frac{\mathrm{d} V}{\mathrm{~d} x} \gg 1,
$$

the term proportional $V(x)$ in (16) can be neglected. The condition (17) corresponds to sufficiently large turbulent magnetic Reynolds numbers. With (17), Eq. (16) transforms into the equation with constant coefficients, and the fundamental eigenfunction satisfying zero boundary conditions is simply $\hat{\beta}(x)=\sin (\pi x / d)$. In this case, the dispersion equation reads

$\gamma=\frac{K_{y}^{2} \ell_{2}^{4}(\gamma)}{4 \eta_{\mathrm{T}}}\left(\frac{\mathrm{d} V}{\mathrm{~d} x}\right)^{2}-\eta_{\mathrm{T}} K^{2}$

where $K^{2}=\pi^{2} / d^{2}+K_{y}^{2}$. The first term on the RHS describes the destabilising effect of shear, and the second term is associated with turbulent dissipation.

The solution of (18) highly depends on the length scales $\ell_{1}$ and $\ell_{2}$ and, hence, on the frequency dependence of the spectral function. It is useful to distinguish between two essentially different types of spectra (Rüdiger 1989). The first type ("turbulence" spectrum) does not vanish for $\omega=0$ and decreases with increasing frequency. For this spectrum, the eddy diffusivity can reach a very large value. As a representative of the turbulence type spectrum we shall use

$$
q(\boldsymbol{k}, \omega)=\frac{Q(\boldsymbol{k})}{\omega^{2}+\tau_{\mathrm{corr}}^{-2}}
$$

where the function $Q$ depends on $\boldsymbol{k}$ alone. The second type of spectrum is vanishing at $\omega \rightarrow 0$ and characterises, for example, an ensemble of "random waves". For this type of spectrum, one can usually use the simplest representation of the frequency dependence,

$q(\boldsymbol{k}, \omega) \propto Q(\boldsymbol{k}) \delta\left(\omega-1 / \tau_{\text {corr }}\right)$,

where $\tau_{\text {corr }}$ is the characteristic time scale of the waves.

\subsection{Random waves}

Substituting the spectral function (20) into (14), we find

$\ell_{1}^{2}=\frac{\ell_{w}^{2}}{1+\gamma^{2} \tau_{\text {corr }}^{2}}, \quad \ell_{2}^{2}=\ell_{w}^{2} \frac{1-\gamma^{2} \tau_{\text {corr }}^{2}}{\left(1+\gamma^{2} \tau_{\text {corr }}^{2}\right)^{2}}$ 


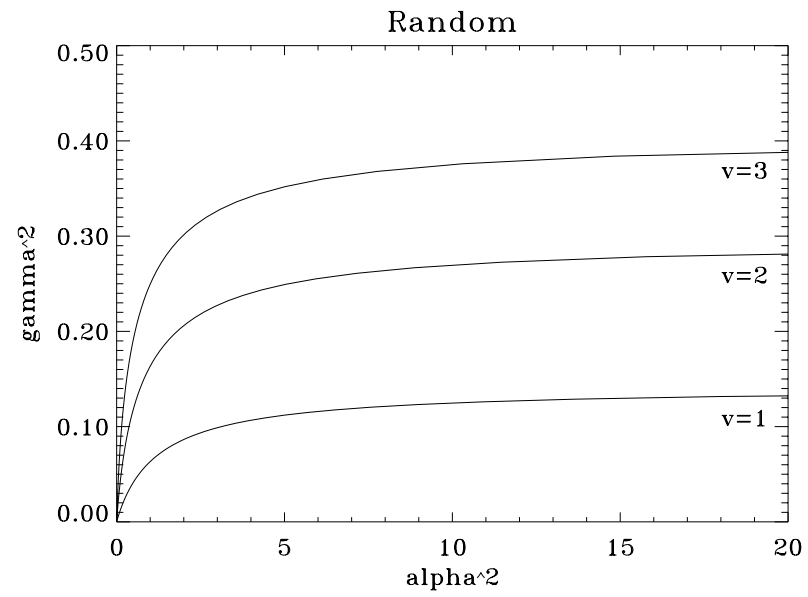

Fig. 1. Solution of dispersion relation (18) for random wave spectrum. The growth rates of the instability for all normalized wave numbers $\alpha=K_{y} d / \pi$ (in flow-direction). $v$ is the normalized shear, $v=\tau_{\text {corr }} \mathrm{d} V / \mathrm{d} x$

where $\ell_{w}=\left(2 / 9 \int Q(\boldsymbol{k}) \mathrm{d} \boldsymbol{k}\right)^{1 / 2} \tau_{\text {corr }}$ is the turbulent length scale. Note that $\ell_{w}>\ell_{1}$ and $\ell_{w}>\ell_{2}$ for $\gamma \neq 0$. For small shear, we can restrict ourselves in linear terms in $\gamma$, then $\ell_{1} \approx \ell_{2} \approx \ell_{w}$. In this case, the dispersion Eq. (18) reduces to

$\gamma^{2}=\frac{K_{y}^{2} \ell_{w}^{2}}{4\left(1+\ell_{w}^{2} K^{2}\right)}\left(\frac{\mathrm{d} V}{\mathrm{~d} x}\right)^{2}$.

Obviously, one of the solutions of this equation corresponds to an unstable dynamo mode. The instability arises for any shear and covers a wide range of wavelengths. Waves with the wavelength much longer than the turbulent length scale, $K \ell_{w} \ll 1$, grow on a time scale $\sim 1 / K \ell_{w} \mathrm{~d} V / \mathrm{d} x$, long compared to the shear time scale, $\mathrm{d} x / \mathrm{d} V$. The growth rate $\gamma$ decreases with decreasing wavelength, reaching the value $\mathrm{d} V / \mathrm{d} x$ for waves with $K \ell_{w} \sim 1$ (Fig. 1).

\section{2. "Turbulence" spectra}

For the spectral function (19), integrations in (14) can be performed by making use of the expressions given by Rüdiger (1989). Then, we have

$\ell_{1}^{2}=\frac{\ell_{\mathrm{T}}^{2}}{\tau_{\text {corr }} \gamma\left(1+\gamma \tau_{\text {corr }}\right)}, \quad \ell_{2}^{2}=-\frac{\ell_{\mathrm{T}}^{2}}{\left(1+\gamma \tau_{\text {corr }}\right)^{2}}$,

where $\ell_{\mathrm{T}}^{2}=2 / 9 \pi \tau_{\text {corr }}^{3} \int Q(\boldsymbol{k}) \mathrm{d} \boldsymbol{k}$. Substituting these expressions into the dispersion Eq. (18), we can rewrite this equation as

$$
\begin{aligned}
& \tau_{\text {corr }} \gamma\left(1+\gamma \tau_{\text {corr }}\right)^{3}= \\
& \ell_{\mathrm{T}}^{2}\left(\frac{1}{4} K_{y}^{2} \tau_{\text {corr }}^{2}\left(\frac{\mathrm{d} V}{\mathrm{~d} x}\right)^{2}-K^{2}\left(1+\gamma \tau_{\text {corr }}\right)^{2}\right)
\end{aligned}
$$

This equation describes four essentially different modes of a mean field which generally can exist in a turbulent fluid

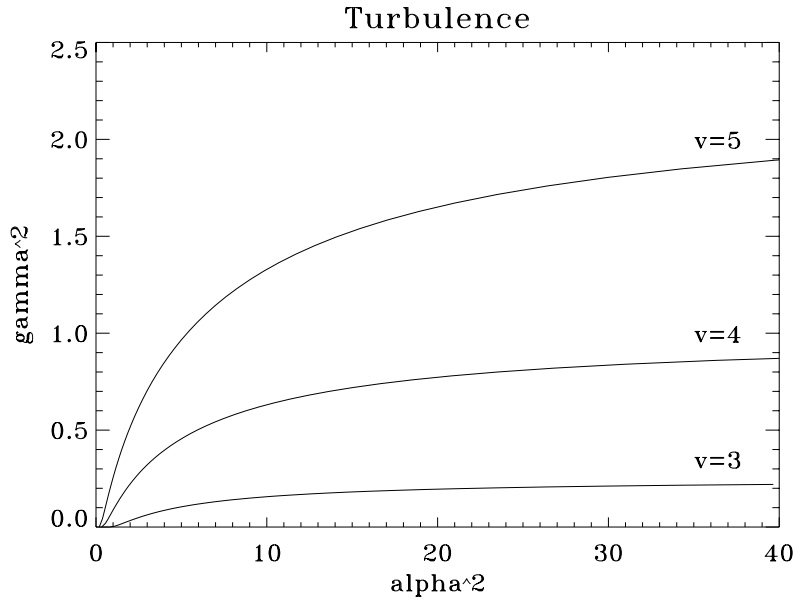

Fig. 2. The same as in Fig. 1 but for spectra of turbulence-type

in the presence of shear. For the mode with $\gamma \ll 1 / \tau_{\text {corr }}$, Eq. (24) yields

$\gamma=\frac{\ell_{\mathrm{T}}^{2}}{\tau_{\text {corr }}}\left(\frac{1}{4} K_{y}^{2} \tau_{\text {corr }}^{2}\left(\frac{\mathrm{d} V}{\mathrm{~d} x}\right)^{2}-K^{2}\right)$.

Instability of dynamo waves arises but only for strong shear rates. The solution of the dispersion relation (24) is given in Fig. 2. Positive growth rates always exist for

$\left|\tau_{\text {corr }} \frac{\mathrm{d} V}{\mathrm{~d} x}\right|>2$.

As in the case of a "random wave" spectrum, the growth rate of instability is higher for waves with a shorter wavelength. However, condition (26) is rather restrictive. For Kepler disks it turns into

$\tau_{\text {corr }}>2 \tau_{\text {rot }} / \pi$,

where $\tau_{\text {rot }}$ is the Keplerian period.

\section{Conclusion}

We have considered the turbulent dynamo action in a shear-flow turbulence. The principal result is that, in the presence of shear, even isotropic and homogeneous turbulence becomes unstable to the generation of a mean magnetic field. The mean field amplification is caused by additional terms which appear in the mean EMF and are proportional to the product of spatial derivatives of the magnetic field and shear stresses. This generation mechanism is qualitatively different to the conventional alphadynamo which apart from a basic rotation requires also a large-scale stratification of turbulence.

In the present paper, we did not use the two-scale model which assumes that the length scale of turbulence is much smaller than that of mean quantities. As a result, we obtain the integral nonlocal equation governing the mean magnetic field. In the case of a linear shear and isotropic and homogeneous turbulence, this equation has a solution corresponding to dynamo waves. The stability properties 
of these waves depend basically on the spectrum of hydrodynamic fluctuations. It turns out that the dynamo waves are always unstable if turbulence has a random wave-type spectrum which results in a very low eddy magnetic diffusivity. If the spectrum of fluctuations is nonvanishing at $\omega \rightarrow 0$ (turbulence-type spectrum) then the instability can arise only for a rather strong shear because this spectrum leads to a high turbulent magnetic diffusivity which stabilises the dynamo waves.

The mechanism considered may play an important role in the generation of magnetic fields in various astrophysical bodies where hydrodynamic flows are characterised by a strong shear, such as in accretion disks, galaxies and jets. As is standard in the dynamo theory, a certain threshold value (here of the normalized shear) must be exceeded for dynamo excitation. In contrast, however, to dynamos working with some alpha-effect, the non-alpha dynamo discussed here will never exhibit steady and global solutions. All shear-flow dynamos should therefore exhibit a much more chaotic behaviour ("flickering"), working on basically smaller scales than the usually considered convection-based dynamos.

Acknowledgements. This research was supported by the Deutsche Forschungsgemeinschaft and the Russian Foundation of Basic Research

\section{Appendix}

\section{A. Magnetic fluctuations}

It is convenient to start with transformations in the $y$ - and $z$-coordinates and to obtain the equation for the quantity

$\hat{\boldsymbol{b}}_{1}\left(x, k_{y}, k_{z}, t\right)=\frac{1}{(2 \pi)^{2}} \int \mathrm{e}^{i\left(k_{y} y+k_{z} z\right)} \boldsymbol{B}^{\prime}(\boldsymbol{r}, t) \mathrm{d} y \mathrm{~d} z$,

with the hat labels as the corresponding Fourier amplitude. Then, we represent $\hat{\boldsymbol{b}}_{1}\left(x, k_{y}, k_{z}, t\right)$ as

$\hat{\boldsymbol{b}}_{1}\left(x, k_{y}, k_{z}, t\right)=\mathrm{e}^{i k_{y} V(x) t} \hat{\boldsymbol{b}}\left(x, k_{y}, k_{z}, t\right)$.

The resulting equation for $\hat{\boldsymbol{b}}\left(x, k_{y}, k_{z}, t\right)$ does not contain the advective term. This equation can easily be solved by Fourier transformation in $x$ and $t$. The solution is

$\hat{\boldsymbol{b}}(\boldsymbol{k}, \omega)=-\frac{i}{\omega_{1}} \hat{\boldsymbol{A}}(\boldsymbol{k}, \omega)-\frac{1}{\omega_{1}^{2}} \frac{\mathrm{d} V}{\mathrm{~d} x} \hat{A}_{x}(\boldsymbol{k}, \omega) \boldsymbol{e}_{y}$

where $\omega_{1}=\omega-i 0 ; \pm 0$ denotes a positive (or negative) contribution of small dissipative terms proportional to $\eta$. The Fourier transformation of $\hat{\boldsymbol{A}}$ is given by

$\hat{\boldsymbol{A}}=\frac{1}{(2 \pi)^{4}} \int \mathrm{e}^{i \boldsymbol{k} \boldsymbol{r}-i\left(\omega+k_{y} V\right) t} \boldsymbol{A}^{\prime}(r, t) \mathrm{d} \boldsymbol{r} \mathrm{d} \boldsymbol{t}$.

\section{$B$. The electromotive force}

Insertion of (6) into (4) yields

$$
\begin{aligned}
& \mathcal{E}=\frac{i}{3} \int \frac{\mathrm{d} \omega^{\prime} \mathrm{d} \boldsymbol{k}^{\prime}}{(2 \pi)^{4}} \frac{\mathrm{d} \omega \mathrm{d} \boldsymbol{k}}{\omega_{1}} \mathrm{~d} \boldsymbol{r}^{\prime} \mathrm{d} t^{\prime} q\left(\boldsymbol{k}^{\prime}, \omega^{\prime}\right) \mathrm{e}^{i\left(\omega+\omega^{\prime}\right)\left(t-t^{\prime}\right)} \\
& \times \mathrm{e}^{i\left(\boldsymbol{k}+\boldsymbol{k}^{\prime}\right)\left(\boldsymbol{r}^{\prime}-\boldsymbol{r}\right)+i k_{y}\left[V(x) t-V\left(x^{\prime}\right) t^{\prime}\right]}\left(\boldsymbol{N}_{1}+\frac{i}{\omega_{1}} \frac{\mathrm{d} V}{\mathrm{~d} x} \boldsymbol{e}_{y} \times \boldsymbol{N}_{2}\right)
\end{aligned}
$$

where

$\boldsymbol{N}_{1}=\nabla^{\prime} \times \overline{\boldsymbol{B}}-\epsilon_{i j k} \frac{k_{j}^{\prime} k_{m}^{\prime}}{k^{\prime 2}} \frac{\partial \bar{B}_{k}}{\partial x_{m}^{\prime}} \boldsymbol{e}_{i}$,

$\boldsymbol{N}_{2}=\nabla^{\prime} \bar{B}_{x}-\frac{k_{m}^{\prime}}{k^{\prime 2}} \frac{\partial \bar{B}_{x}}{\partial x_{m}^{\prime}} \boldsymbol{k}^{\prime}-i\left(\boldsymbol{k}^{\prime} \cdot \overline{\boldsymbol{B}}\right)\left(\boldsymbol{e}_{x}-\boldsymbol{k}^{\prime} \frac{k_{x}^{\prime}}{k^{\prime 2}}\right)$,

with $\overline{\boldsymbol{B}}=\overline{\boldsymbol{B}}\left(\boldsymbol{r}^{\prime}, t^{\prime}\right)$; summation is over repeated indexes.

Introducing the new wave vector $\boldsymbol{k}^{\prime \prime}=\boldsymbol{k}+\boldsymbol{k}^{\prime}$ instead of $\boldsymbol{k}$, the integrals over $\mathrm{d} \boldsymbol{k}$ and $\mathrm{d} \omega$ in (32) can be transformed as in

$$
\begin{array}{r}
\int \frac{\mathrm{d} \omega \mathrm{d} \boldsymbol{k}}{\omega_{1}^{\nu}} \mathrm{e}^{i\left(\omega+\omega^{\prime}\right)\left(t-t^{\prime}\right)-i\left(\boldsymbol{k}+\boldsymbol{k}^{\prime}\right) \cdot\left(\boldsymbol{r}-\boldsymbol{r}^{\prime}\right)+i k_{y}\left[V(x) t-V\left(x^{\prime}\right) t^{\prime}\right]} \\
=\mathrm{e}^{-i \boldsymbol{k}^{\prime}\left[\boldsymbol{V}(x) t-\boldsymbol{V}\left(x^{\prime}\right) t^{\prime}\right]} \int \mathrm{d} \boldsymbol{k}^{\prime \prime} \mathrm{e}^{i \boldsymbol{k}^{\prime \prime} \cdot\left[\boldsymbol{r}^{\prime}-\boldsymbol{r}+\boldsymbol{V}(x) t-\boldsymbol{V}\left(x^{\prime}\right) t^{\prime}\right]} \\
\times \int \frac{\mathrm{d} \omega \mathrm{e}^{i \omega\left(t-t^{\prime}\right)}}{\left(\omega-\omega^{\prime}-i 0\right)^{\nu}} .
\end{array}
$$

Integrals over $\mathrm{d} \omega$ can be reduced to the known integrals

$$
\int_{-\infty}^{+\infty} \frac{\mathrm{e}^{-i p x} \mathrm{~d} x}{(i x+\beta)^{\nu}}= \begin{cases}0 & \text { if } p>0 \\ 2 \pi[\Gamma(\nu)]^{-1}(-p)^{\nu-1} \mathrm{e}^{\beta p} & \text { if } p<0\end{cases}
$$

with the gamma-function $\Gamma ; \operatorname{Re} \nu>0, \operatorname{Re} \beta>0$. Substituting the value of these integrals into (32), we obtain

$$
\begin{aligned}
& \mathcal{E}=\frac{1}{3} \int \mathrm{d} \omega^{\prime} \mathrm{d} \boldsymbol{k}^{\prime} q\left(\boldsymbol{k}^{\prime}, \omega^{\prime}\right) \int_{-\infty}^{t} \mathrm{~d} t^{\prime} \mathrm{d} \boldsymbol{r}^{\prime} \mathrm{e}^{i \omega^{\prime}\left(t-t^{\prime}\right)} \int \frac{\mathrm{d} \boldsymbol{K}}{(2 \pi)^{3}} \\
& \times \mathrm{e}^{i \boldsymbol{k}^{\prime \prime}\left[\boldsymbol{r}^{\prime}-\boldsymbol{r}+\boldsymbol{V}(x) t-\boldsymbol{V}\left(x^{\prime}\right) t^{\prime}\right]}\left(\left(t-t^{\prime}\right) \frac{\mathrm{d} V}{\mathrm{~d} x} \boldsymbol{e}_{y} \boldsymbol{N}_{2}-\boldsymbol{N}_{1}\right) .
\end{aligned}
$$

The integral over $\mathrm{d} \boldsymbol{k}^{\prime \prime}$ yields one of the representations of the $\delta$-function. Since $\boldsymbol{V}$ is parallel to the $y$-axis, the argument of the $\delta$-function can easily be transformed to $\left(\boldsymbol{r}^{\prime}-\boldsymbol{r}+\boldsymbol{V}(x)\left(t-t^{\prime}\right)\right)$. Then, integrating over $\mathrm{d} \boldsymbol{r}^{\prime}$, for the mean EMF we arrive at

$$
\begin{aligned}
& \mathcal{E}=-\frac{1}{3} \int \mathrm{d} \omega^{\prime} \mathrm{d} \boldsymbol{k}^{\prime} q\left(\boldsymbol{k}^{\prime}, \omega^{\prime}\right) \int_{-\infty}^{t} \mathrm{~d} t^{\prime} \mathrm{e}^{i \omega^{\prime}\left(t-t^{\prime}\right)} \\
& \times\left.\left(\boldsymbol{N}_{1}\left(\boldsymbol{r}^{\prime}, t^{\prime}\right)-\left(t-t^{\prime}\right) \frac{\mathrm{d} V}{\mathrm{~d} x} \boldsymbol{e}_{y} \times \boldsymbol{N}_{2}\left(\boldsymbol{r}^{\prime}, t^{\prime}\right)\right)\right|_{\boldsymbol{r}^{\prime}=\boldsymbol{r}-\boldsymbol{V}\left(t-t^{\prime}\right)} .
\end{aligned}
$$

Since according to our assumption, turbulence is locally isotropic and homogeneous, we can average $\boldsymbol{N}_{1}$ and $\boldsymbol{N}_{2}$ over directions of the vector $\boldsymbol{k}$ and obtain $\boldsymbol{N}_{1}=2 / 3 \operatorname{rot} \overline{\boldsymbol{B}}$ and $\boldsymbol{N}_{2}=2 / 3 \operatorname{grad} \bar{B}_{x}$. 


\section{Slow-flow approximation}

The RHS of Eq. (11) contains integrals of the type

$I=\int_{0}^{\infty} F(\tau) a(\tau) \mathrm{e}^{i K_{y} V(x) \tau} \mathrm{d} \tau$,

where $a(\tau)=\mathrm{e}^{-\gamma \tau}$ or $\tau \mathrm{e}^{-\gamma \tau}$. Substituting $F(\tau)$, we can transform these integrals as

$I=\frac{2}{9} \int_{0}^{\infty} \int_{0} a(\tau) q\left(\boldsymbol{k}, \omega-K_{y} V\right) \mathrm{e}^{i \omega \tau} \mathrm{d} \tau \mathrm{d} \boldsymbol{k} \mathrm{d} \omega$.

If we consider the waves with a long wavelength in the flow direction $y$, i.e. $\tau_{\text {corr }} V K_{y} \ll 1$, then we can expand the spectral function in a power series in this small parameter. Restricting ourselves to the lowest order, we have

$I \approx \frac{2}{9} \int_{0}^{\infty} \int^{\infty} q(\boldsymbol{k}, \omega) a(\tau) \mathrm{e}^{i \omega \tau} \mathrm{d} \tau \mathrm{d} \boldsymbol{k} \mathrm{d} \omega$.

\section{References}

Aleksandrov, A., Kolmogorov, A., \& Laurentiev, M. 1963, Mathematics: Its Content, Methods, and Meaning (MIT Press)

Blackman, E. G. 2000, ApJ, 529, 138

Brandenburg, A., \& Urpin, V. 1998, A\&A, 332, L41

Hoyng, P. 1985, J. Fluid Mech., 151, 295

Krause, F., \& Rädler, K.-H. 1980, Mean-field magnetohydrodynamics and dynamo theory (Akademie-Verlag, Berlin)

Moffatt, H. K. 1978, Magnetic field generation in electrically conducting fluids (Cambridge University Press, Cambridge)

Parker, E. N. 1979, Cosmical magnetic fields (Clarendon Press, Oxford)

Pipin, V., Rüdiger, G., \& Elstner, D. 2001, GAFD (in preparation)

Rüdiger, G. 1989, Differential rotation and stellar convection (Gordon \& Breach, New York)

Urpin, V. 1999a, A\&A, 347, L47

Urpin, V. 1999b, MNRAS, 308, 741 